Editor's Note: These short, critical reviews of recent papers in the Journal, written exclusively by graduate students or postdoctoral fellows, are intended to summarize the important findings of the paper and provide additional insight and commentary. For more information on the format and purpose of the Journal Club, please see http://www.jneurosci.org/misc/ifa_features.shtml.

\title{
Transcription Factor Lim1 Specifies Horizontal Cell Laminar Position in the Retina
}

\author{
Milica A. Margeta \\ Neurosciences Program, Stanford University, Stanford, California 94305-5020 \\ Review of Poché et al. (http://www.jneurosci.org/cgi/content/full/27/51/14099)
}

The vertebrate retina is a highly laminated structure, with seven classes of cells organized in a number of discrete layers. Although much progress has been made in understanding early developmental events that specify differentiation of retinal cell subtypes (for review, see Hatakeyama and Kageyama, 2004), factors that regulate correct laminar positioning and connectivity of the retina remain poorly understood. A recent study has implicated adhesion molecules Sidekicks and Dscam in the development of correct synaptic connections in the sublaminae of the inner plexiform layer (Yamagata and Sanes, 2008). However, less is known about the "intermediate" laminar specifiers: molecules that act to properly position cells within the correct retinal layer during development, thus enabling subsequent circuit formation.

A recent study by Poché et al. (2007) identifies just such a factor. In an elegant series of experiments, the authors analyze the role of the LIM class homeodomain transcription factor, Lim1 (Lhx1), in correct laminar positioning of retinal horizontal cells. Horizontal cells (HCs) are

Received Feb. 5, 2008; revised Feb. 22, 2008; accepted Feb. 23, 2008.

This work was supported by the Stanford Medical Scientist Training Program and National Institutes of Health Grant GM007365. I thank G. Maro and M. Patel for thoughtful comments on this manuscript.

Correspondence should be addressed to Milica A. Margeta, Neurosciences Program, Stanford University, Stanford, CA 94305-5020. E-mail: mmargeta@stanford.edu.

D0I:10.1523/JNEUROSCI.0535-08.2008

Copyright $\odot 2008$ Society for Neuroscience $\quad 0270-6474 / 08 / 283835-02 \$ 15.00 / 0$ retinal interneurons that modulate neurotransmission between the photoreceptors and bipolar cells. Past studies have identified a number of transcription factors required for the specification of the HC fate (Hatakeyama and Kageyama, 2004). However, these studies do not address the question of how differentiating HCs, which migrate bidirectionally throughout the developing neuroepithelium (Edqvist and Hallbook, 2004), eventually localize to the correct layer in the retina.

The first hint that Lim 1 may be important for HC positioning came from its expression pattern. Whereas $\operatorname{Lim} 1$ is widely expressed in the developing and adult CNS, its expression pattern in the retina is restricted to differentiating and mature HCs [Poché et al. (2007), their Fig. 1A-H (http://www.jneurosci.org/cgi/content/full/ 27/51/14099/F1)]. Importantly, Lim1 expression first becomes noticeable at embryonic day 14.5 (E14.5), when HC genesis is largely complete, suggesting that Lim1 labels differentiating, postmitotic HCs. The authors further confirm this observation by costaining embryonic retinal sections for Lim1 and a cell cycle marker, Ki67, and finding no overlap between these two markers [Poché et al. (2007), their Fig. 1 I-L (http://www.jneurosci. org/cgi/content/full/27/51/14099/F1)]. This result suggests that Lim1 does not play a role in the $\mathrm{HC}$ fate determination.

To assess the role of Lim 1 in HC development, Poché et al. (2007) made a retina-specific Lim1 knock-out using a Lim-1 ${ }^{\text {flox }}$ allele together with retinaspecific Cre-expressing lines. In combination with this conditional allele, the authors used a $\operatorname{Lim}-1^{\text {lac-Z }}$ null allele to trace Lim-1-expressing cells. In the mutant Lim-1 retinas, lacZ + cells were not restricted to the outer plexiform layer, as is seen in the wild-type situation. Instead, a large proportion of lacZ + cells was observed to project into the inner plexiform layer and stratify adjacent to the ganglion cell layer [Poché et al. (2007), their Fig. 2A-F (http://www.jneurosci.org/cgi/ content/full/27/51/14099/F2)]. This kind of stratification pattern is characteristic of amacrine cells, and indeed, the authors find that the Lim-1 mutant cells adopt features of amacrine cell morphology [Poché et al. (2007), their Fig. 2G-I (http://www. jneurosci.org/cgi/content/full/27/51/ 14099/F2)].

Because the ectopic lacZ+ cells localize to the amacrine cell layer and take on amacrine-like morphology, one might wonder whether their molecular identity has also been disrupted. The authors address this question with an extensive panel of markers that have traditionally been used to characterize HCs and other cellular populations within the retina. The authors find that the ectopic lacZ + cells display a molecular "signature" that is consistent only with the HC fate [Poché et al. (2007), their Figs. 3, 5 (http://www. jneurosci.org/cgi/content/full/27/51/14099/ F3) (http://www.jneurosci.org/cgi/content/ 
full/27/51/14099/F5)], whereas they do not express markers characteristic of amacrine cells or any other retinal subtypes [Poché et al. (2007), their Fig. 4 (http:// www.jneurosci.org/cgi/content/full/27/ 51/14099/F4) and Table 1 (http://www. jneurosci.org/cgi/content/full/27/51/ 14099/T1)]. Together, these results strongly suggest that the ectopic LacZ+ cells are indeed horizontal cells and that their pseudoamacrine morphology is not a result of a cell-fate switch but may be environmentally determined.

To gain more insight into the mechanism by which Lim 1 affects HC positioning, the authors performed a time course of the mutant HC migration [Poché et al. (2007), their Fig. 6 (http://www. jneurosci.org/cgi/content/full/27/51/ 14099/F6)]. The first misplaced cells are initially observed at E15.5, and by E18.5, a clear failure of lacZ+ cells to migrate to the outer neuroblastic layer (onbl) is evident. Based on these findings, the authors propose a model illustrated in their Figure 7 (http://www.jneurosci. org/cgi/content/full/27/51/14099/F7). Normally, developing HCs are born in the onbl, migrate to the inner neuroblastic layer (inbl) in which Lim1 expression is turned on, and subsequently migrate back to their proper position, characterized by the dendritic arborization in the outer plexiform layer. In the mutant cells, the absence of $\operatorname{Lim} 1$ expression causes them to be retained in the inbl, leading to the arborization in the inner plexiform layer and amacrine-like morphology.

The pseudoamacrine morphology of ectopic HC cells is an interesting finding, suggesting that the molecular identity of a cell and its morphology can be genetically separated. This conclusion of morphological "plasticity" would be strengthened by more rigorous anatomical and electrophysiological studies of the mispositioned lacZ+ cells. Furthermore, it would be interesting to know what the consequences are of HC "mislaminization" on the visual acuity and processing in the mutant mice. Finally, one could perform a gain-offunction experiment by ectopically expressing Lim 1 and seeing whether this is sufficient to localize other retinal cell types to the HC layer.

Many questions remain in terms of understanding the mechanism of Lim1 action. What are the downstream targets of Lim 1 that mediate the HC migration to the proper retinal layer? What is the nature of signals that tell wild-type HCs to stop migrating at the proper position in the onbl, and conversely, what is the nature of signals that keep mutant HCs "stuck" in the inbl? Finally, Lim1 continues to be expressed in HCs long after their migration to the proper retinal layer has been achieved. What is the role of Lim 1 in mature $\mathrm{HCs}$, and is it responsible for maintaining HC position, morphology, or function?

Overall, the study by Poché et al. (2007) is the first study clearly implicating a transcription factor in retinal lamination. Given that a number of other Lhx family members are expressed in the retina (Zhang et al., 2006), it is tempting to speculate that there exists an LIM transcription factor code specifying lamination throughout the retina and possibly elsewhere in the brain.

\section{References}

Edqvist PH, Hallbook F (2004) Newborn horizontal cells migrate bidirectionally across the neuroepithelium during retinal development. Development 131:1343-1351.

Hatakeyama J, Kageyama R (2004) Retinal cell fate determination and bHLH factors. Semin Cell Dev Biol 15:83-89.

Poché RA, Kwan KM, Raven MA, Furuta Y, Reese $\mathrm{BE}$, Behringer RR (2007) Liml is essential for the correct laminar positioning of retinal horizontal cells. J Neurosci 27:14099-14107.

Yamagata M, Sanes JR (2008) Dscam and Sidekick proteins direct lamina-specific synaptic connections in vertebrate retina. Science 451:465-469.

Zhang SS, Xu X, Liu MG, Zhao H, Soares MB, Barnstable CJ, Fu XY (2006) A biphasic pattern of gene expression during mouse retina development. BMC Dev Biol 6:48. 